\title{
Analyticity of the Scattering Operator for the Nonlinear Klein-Gordon Equation with Cubic Nonlinearity
}

\section{Peter Kumlin}

Department of Mathematics, Chalmers University of Technology, S-41296 Göteborg, Sweden

Received January 7, 1991; in revised form April 19, 1991

Abstract. The wave and scattering operators for the equation

$$
\left(\square+m^{2}\right) \varphi+\lambda \varphi^{3}=0
$$

with $m>0$ and $\lambda>0$ on four-dimensional Minkowski space are analytic on the space of finite-energy Cauchy data, i.e. $L_{2}^{1}\left(\mathbf{R}^{3}\right) \oplus L_{2}\left(\mathbf{R}^{3}\right)$.

\section{Introduction}

This paper answers a question, raised by Baez and Zhou in [2], whether the scattering and wave operators for

$$
\left(\square+m^{2}\right) \varphi+\lambda \varphi^{3}=0, \quad m>0, \quad \lambda>0,
$$

are analytic or not on the whole space of finite-energy data $L_{2}^{1}\left(\mathbf{R}^{3}\right) \oplus L_{2}\left(\mathbf{R}^{3}\right)$. The answer is affirmative. This implies, which is noted in [2], that the massive $\varphi^{4}$ theory is completely integrable. The same holds for the massless $\varphi^{4}$ theory proved by Baez in [1].

We start by introducing some notation and some basic facts following the presentation given in [2]. Consider Eq. (1), where $\varphi=\varphi(t, x)$ is a realvalued function on $\mathbf{R} \times \mathbf{R}^{3}$. Let $L_{q}^{s}\left(\mathbf{R}^{3}\right)$ denote the Sobolev space of functions on $\mathbf{R}^{3}$ with $s$ derivatives in $L_{q}$ and let $\mathbf{X}$ denote the Hilbert space $L_{2}^{1}\left(\mathbf{R}^{3}\right) \oplus L_{2}\left(\mathbf{R}^{3}\right)$ with norm $\|\cdot\|_{X}$ given by

$$
\left\|\left(u_{1}, u_{2}\right)\right\|_{\mathbf{X}}=\left(\frac{1}{2} \int_{\mathbf{R}^{3}}\left(\left|\nabla u_{1}\right|^{2}+m^{2} u_{1}^{2}+u_{2}^{2}\right) d x\right)^{1 / 2} .
$$

Given $u \in \mathbf{X}$ there is a unique distributional solution $\varphi$ of (1) with

$$
\left.(\varphi, \dot{\varphi})\right|_{t=0}=u \text {. }
$$

Let $U(t) u=\left.(\varphi, \dot{\varphi})\right|_{t}$ and let $U_{0}(t)$ be the orthogonal linear operator on $\mathbf{X}$ corresponding to the case $\lambda=0$, i.e. the linear Klein-Gordon equation. If $N\left(u_{1}, u_{2}\right)=$ 
$\left(0,-\lambda u_{1}^{3}\right)$, which maps $\mathbf{X}$ into $\mathbf{X}$, then $U$ may be defined in terms of $U_{0}$ :

$$
U(t) u=U_{0}(t) u+\int_{0}^{t} U_{0}(t-s) N(U(s) u) d s .
$$

For any $u \in \mathbf{X}$, there exist $u_{ \pm} \in \mathbf{X}$ such that

$$
\left\|U_{0}(t) u_{ \pm}-U(t) u\right\|_{\mathbf{X}} \rightarrow 0 \text { as } t \rightarrow \pm \infty .
$$

In fact there are homeomorphisms, the wave operators,

$$
W_{ \pm}: \mathbf{X} \rightarrow \mathbf{X}
$$

such that $W_{ \pm}\left(u_{ \pm}\right)=u$. The scattering operator

$$
S: \mathbf{X} \rightarrow \mathbf{X}
$$

is defined by $S\left(u_{-}\right)=u_{+}$, i.e. $S=\left(W_{+}\right)^{-1} W_{-}$. The existence of $S$ for low energy is due to Strauss [12] and on the whole space of finite energy to Brenner [4].

To state our results we need some more notation. Let $L_{p}\left(L_{q}^{s}\right)$ denote $L_{p}\left(\mathbf{R} ; L_{q}^{s}\left(\mathbf{R}^{3}\right)\right)$ and set $\mathbf{Z}=L_{\infty}\left(L_{1}^{2}\right) \cap L_{3}\left(L_{6}\right)$ with norm

$$
\|\varphi\|_{\mathbf{Z}}=\|\varphi\|_{L_{x}\left(L_{2}^{1}\right)}+\|\varphi\|_{L_{3}\left(L_{6}\right)} .
$$

Furthermore by an analytic mapping we mean an infinitely Frechét differentiable mapping with a locally norm-convergent Taylor series. Our main result is

Theorem 1.1. The operators $W_{ \pm}$and $S$ are analytic from $\mathbf{X}$ to $\mathbf{X}$.

The proof of Theorem 1.1 rests on the following result.

Theorem 1.2. Let $u \in \mathbf{X}$ and let $\varphi=\varphi(u)$ be the unique distributional solution of (1) with $u=\left.(\varphi, \dot{\varphi})\right|_{t=0}$. Then $\varphi \in \mathbf{Z}$ and the mapping $u \mapsto \varphi$ is analytic from $\mathbf{X}$ to $\mathbf{Z}$.

Theorem 1.2 is a generalization of Theorem 1 in [2] and follows from an application of an implicit function theorem. We use the same approach here, but where Baez and Zhou argue with the contraction mapping principle we use a Fredholm theory argument. The crucial fact for our method to work is that a certain operator is power-compact (the main lemma).

This paper is organized as follows: Sect. 2 contains a proof of Theorem 1.2, Sect. 3 contains a proof of the main lemma and Sect. 4 contains a proof of Theorem 1.1.

\section{Proof of Theorem 1.2}

The existence of the distributional solution $\varphi$ of (1) and $\varphi \in \mathbf{Z}$ is proved in [2], so it only remains to show that the mapping $u \mapsto \varphi(u)$ is analytic. For $u \in \mathbf{X}$ and $\psi \in \mathbf{Z}$ consider the mapping

$$
R(u, \psi):=\varphi_{0}(u)-\lambda \int_{0}^{t} K(t-s) \psi^{3}(s) d s-\psi(t),
$$

where

$$
K(t):=\left(\sqrt{-\Delta+m^{2}}\right)^{-1} \sin \left(t \sqrt{-\Delta+m^{2}}\right)
$$


and $\varphi_{0}(u)$ denotes the solution of the linear Klein-Gordon equation

$$
\left(\square+m^{2}\right) \varphi=0
$$

with data $\left.(\varphi, \dot{\varphi})\right|_{t=0}=u$. The following lemma we quote from [2].

Lemma 2.1. Given $\varphi_{1}, \varphi_{2}, \varphi_{3} \in \mathbf{Z}$, let

$$
\psi(t)=\int_{0}^{t} K(t-s) \varphi_{1}(s) \varphi_{2}(s) \varphi_{3}(s) d s .
$$

Then $\psi \in \mathbf{Z}$ and the map $\left(\varphi_{1}, \varphi_{2}, \varphi_{3}\right) \mapsto \psi$ is analytic from $\mathbf{Z}^{3}$ to $\mathbf{Z}$.

This and Lemma 3.2 below imply that $R$ maps $\mathbf{X} \times \mathbf{Z}$ into $\mathbf{Z}$. Moreover $R: \mathbf{X} \times$ $\mathbf{Z} \rightarrow \mathbf{Z}$ is analytic, since it is linear in $u$ and multilinear in $\psi$ (Hartogs theorem). Finally, $R(u, \varphi(u))=0$, since $R(u, \varphi)=0$ is the integralequational formulation of (1) with data (2). The analyticity of $u \mapsto \varphi(u)$ is, according to the analytic implicit function theorem [3], a consequence of the invertibility of

$$
D_{2} R(u, \varphi(u)): \mathbf{Z} \rightarrow \mathbf{Z}
$$

for each $u \in \mathbf{X}$, where $D_{2}$ denotes the Frechét derivative in the second argument. We remark that from now on the analyticity plays no rôle. This implies that our method can give $C^{s}$-regularity for $u \mapsto \varphi(u)$ once we can prove that the mapping $R$ from $\mathbf{X} \times \mathbf{Z}$ to $\mathbf{Z}$ is $C^{s}$-regular, provided we can show that $D_{2} R(u, \varphi(u)): \mathbf{Z} \rightarrow \mathbf{Z}$ is invertible for each $u \in \mathbf{X}$. The technique can also be generalized to higher space dimensions choosing appropriate function spaces $\mathbf{Z}$. It is also from here that our argument differs from that of Baez and Zhou, namely they prove the invertibility of $D_{2} R(u, \varphi(u))$ by a contraction argument and are thereby limited to low energy data.

The rest of this section contains a proof of the invertibility of $D_{2} R(u, \varphi(u))$. For $u \in \mathbf{X}$ and $\psi \in \mathbf{Z}$ we have

$$
\left(D_{2} R(u, \varphi(u))(\psi)\right)(t)=-3 \lambda \int_{0}^{t} K(t-s)\left(\varphi(u)^{2} \psi\right)(s) d s-\psi(t) .
$$

To prove the injectivity of $D_{2} R(u, \varphi(u))$ we argue as follows.

Suppose that $D_{2} R(u, \varphi(u)) \psi=0$, so that

$$
\psi(t)=-3 \lambda \int_{0}^{t} K(t-s)\left(\varphi(u)^{2} \psi\right)(s) d s .
$$

Then by Proposition 3.1 below, we obtain that

$$
\begin{aligned}
\|\psi(t)\|_{L_{2}^{1}} & \leqq C \int_{0}^{t}\left(\|\varphi(u)(s)\|_{L_{2}^{1}}\right)^{2}\|\psi(s)\|_{L_{2}^{1}} d s \\
& \leqq C \int_{0}^{t}\|\psi(s)\|_{L_{2}^{1}} d s,
\end{aligned}
$$

where the conservation of energy, i.e.

$$
\|\varphi(u)\|_{L_{\infty}\left(L_{2}^{1}\right)} \leqq C\|u\|_{\mathbf{x}} .
$$

is used. Finally $\psi=0$ follows from Gronwall's inequality. By the open mapping 
theorem it remains to prove that $D_{2} R(u, \varphi(u))$ is surjective. Set

$$
T_{u} \psi(t):=3 \lambda \int_{0}^{t} K(t-s)\left(\varphi(u)^{2} \psi\right)(s) d s .
$$

Then we obtain $D_{2} R(u, \varphi(u))=-\left(I+T_{u}\right)$. Hence, if $T_{u}$ were a compact operator from $\mathbf{Z}$ to $\mathbf{Z}$, then the Fredholm alternative theorem, see [7], would yield surjectively, since $I+T_{u}$ is injective. However, $T_{u}$ is (probably) not compact, but it is enough to show that $T_{u}$ is power-compact, i.e. $T_{u}^{k}$ is compact for some positive integer $k$, e.g. $k=3$. The reason is that we can factor out $I+T_{u}$ from $I+T_{u}^{3}$, i.e. we have

$$
I+T_{u}^{3}=\left(I+T_{u}\right)\left(I-T_{u}+T_{u}^{3}\right)
$$

The Fredholm alternative theorem can still be applied since $I+T_{u}^{3}$ is injective by an analogous argument to the one given above for $I+T_{u}$ using Proposition 3.1. Hence (6) gives the surjectivity of $I+T_{u}$. So it remains to prove

Lemma 2.2 (Main Lemma). Let $u \in \mathbf{X}$. Then

is compact.

$$
T_{u}^{3}: \mathbf{Z} \rightarrow \mathbf{Z}
$$

\section{Proof of Power-Compactness of $T_{u}$}

We immediately conclude from Lemma 2.1 that $T_{u}$ is a bounded linear operator on $\mathbf{Z}$. In particular, we have the simple but crucial estimate

$$
\left\|T_{u} \psi\right\|_{L_{\infty}\left(L_{2}^{1}\right)} \leqq C\|\psi\|_{L_{3}\left(L_{6}\right)},
$$

which follows from Proposition 3.1 and the first part of Theorem 1.2. Hence it is enough to show that $T_{u}^{2}$ is a compact operator from $\mathbf{Z}$ to $L_{3}\left(L_{6}\right)$. Moreover we can argue with sequences since a subset of a Banach space is strongly relatively compact iff it is strongly relatively sequentially compact. (This follows for all metrizable spaces.) Hence it is (more than) enough to prove that for each bounded sequence $\left\{\psi_{j}\right\}_{j=0}^{\infty}$ in $\mathbf{Z}$, the sequence $\left\{T_{u} \psi_{j}\right\}_{j=0}^{\infty}$ has a convergent subsequence in $L_{3}\left(L_{6}\right)$.

The idea of the proof is simply to extract a converging subsequence of $\left\{T_{u} \psi_{j}\right\}_{j=0}^{\infty}$ in $L_{3}\left([-N, N] ; L_{6}(\{|x| \leqq N\})\right)$ for $N=1,2,3, \ldots$ and then show that a Cantor diagonalization process yields a converging subsequence in $L_{3}\left(L_{6}\right)$. The proof is divided into four steps.

Step 1.

$$
\lim _{T \rightarrow \infty} \sup _{j=1,2,3, \ldots}\left\|T_{u} \psi_{j}\right\|_{L_{3}\left((-\infty,-T] \cup[T, \infty) ; L_{6}\right)}=0
$$

Step 2.

$$
\lim _{R \rightarrow \infty} \sup _{j=1,2,3, \ldots}\left\|T_{u} \psi_{j}\right\|_{L_{3}\left([-T, T] ; L_{6}(\{|x| \geqq R\})\right)}=0 \text { for all } T>0 .
$$

Step 3.

$\left\{T_{u} \psi_{j}\right\}$ has a convergent subsequence in $L_{3}\left([-T, T] ; L_{6}(\{|x| \leqq R\})\right)$ for all $R, T>0$.

Step 4.

A Cantor diagonalization process. 
Throughout the paper we let $\varphi$ and $\varphi_{0}$ denote the solutions of (1) with data (2) and (1), for $\lambda=0$, with the same data (2) respectively. When we wish to indicate the dependence on data $u$ we write $\varphi(u)$ and $\varphi_{0}(u)$ respectively. However we use, inconsistently with the rule above, the notation $\varphi(t)$ and $\varphi_{0}(t)$ to indicate the timedependence. It should be clear from the context what is meant. Thus with these notations and with $K$ defined by (3), we have

$$
\varphi(t)=\varphi_{0}(t)-\lambda \int_{0}^{t} K(t-s) \varphi(s)^{3} d s
$$

We now recall some wellknown estimates that will be used repeatedly.

Proposition 3.1. $\left(L_{p}-L_{p^{\prime}}\right.$ estimate). Let $1<p \leqq 2 \leqq p^{\prime}, \frac{1}{p}+\frac{1}{p^{\prime}}=1$ and $\delta:=\frac{1}{2}-\frac{1}{p^{\prime}}$ and $0 \leqq \theta \leqq 1$. Then if $\delta(n+1+\theta) \leqq 1+s-s^{\prime}$,

where

$$
\|K(t) g\|_{L_{p^{\prime}}^{s^{\prime}}} \leqq k(t)\|g\|_{L_{p}^{s}}, \quad t \geqq 0
$$

$$
k(t)=C\left\{\begin{array}{ll}
t^{-(n-1-\theta) \delta}, & 0<t \leqq 1 \\
t^{-(n-1+\theta) \delta}, & 1 \leqq t
\end{array} .\right.
$$

In particular, we will use this estimate with $n=3, p=8 / 7, p^{\prime}=8, s=1$ and $s^{\prime}=0$. We refer to Brenner [4] for a proof.

Furthermore we quote a space-time estimate from [2], that is proved along the lines for similar estimates of Marshall/Strauss/Wainger [10], Marshall [9] and others.

\section{Lemma 3.2.}

$$
\left\|\varphi_{0}\right\|_{\mathbf{z}} \leqq C\|u\|_{\mathbf{x}}
$$

For a proof see [2].

Step 1.

Let $T>0$ be fixed. Hölder's inequality yields

$$
\begin{aligned}
& \int_{T}^{\infty}\left(\left\|\int_{0}^{t} K(t-s)\left(\varphi^{2} \psi(s)\right) d s\right\|_{L_{6}}\right)^{3} d t \\
& \quad \leqq \int_{T}^{\infty}\left(\left\|\int_{0}^{t} K(t-s)\left(\varphi^{2} \psi(s)\right) d s\right\|_{L_{2}}\right)^{3 / 9} \cdot\left(\left\|\int_{0}^{t} K(t-s)\left(\varphi^{2} \psi(s)\right) d s\right\|_{L_{8}}\right)^{24 / 9} d t \\
& \quad \leqq C \int_{T}^{\infty}\left(\int_{0}^{t}\left\|\varphi^{2} \psi(s)\right\|_{L_{2}} d s\right)^{1 / 3} \cdot\left(\int_{0}^{t}\left\|K(t-s)\left(\varphi^{2} \psi(s)\right)\right\|_{L_{8}} d s\right)^{8 / 3} d t \\
& \quad \leqq C \int_{T}^{\infty}\left(\int_{-\infty}^{\infty}\left(\|\varphi(s)\|_{L_{6}}\right)^{2}\|\psi(s)\|_{L_{6}} d s\right)^{1 / 3} \cdot\left(\int_{0}^{t} k(t-s)\left\|\varphi^{2} \psi(s)\right\|_{L_{8 / 7}^{1}} d s\right)^{8 / 3} \\
& \quad \leqq C\left(\|\varphi\|_{\mathrm{Z}}\right)^{2 / 3}\left(\|\psi\|_{\mathrm{Z}}\right)^{1 / 3} \cdot \int_{T}^{\infty}\left(\int_{0}^{t} k(t-s)\left\|\varphi^{2} \psi(s)\right\|_{L_{8 / 7}^{1}} d s\right)^{8 / 3} d t,
\end{aligned}
$$

where we have used Proposition 3.1 with $k$ being a positive $L_{1}$-function. To simplify 
notations set $g(s):=\left\|(\varphi(s))^{2} \psi(s)\right\|_{L_{8 / 7}^{1}}$. Then we obtain with Jensen's inequality

$$
\begin{aligned}
\int_{T}^{\infty}\left(\int_{0}^{t} k(t-s) g(s) d s\right)^{8 / 3} d t & =\int_{T}^{\infty}\left(\int_{0}^{t} k(s) g(t-s) d s\right)^{8 / 3} d t \\
\leqq & \int_{T}^{\infty}\left(\int_{0}^{t} k(s) d s\right)^{5 / 3} \cdot \int_{0}^{t} k(s) g(t-s)^{8 / 3} d s d t \\
\leqq & C \int_{T}^{\infty} \int_{0}^{t} k(s) g(t-s)^{8 / 3} d s d t \\
= & C \int_{T}^{\infty} \int_{0}^{t} k(t-s) g(s)^{8 / 3} d s d t \\
= & C\left(\int_{0}^{T} \int_{T}^{\infty} k(t-s) d t g(s)^{8 / 3} d s+\int_{T}^{\infty} \int_{s}^{\infty} k(t-s) d t g(s)^{8 / 3} d s\right) \\
= & C\left(\int_{0}^{T / 2} \int_{T}^{\infty} k(t-s) d t g(s)^{8 / 3} d s+\int_{T / 2}^{\infty} \int_{T}^{\infty} k(t-s) d t g(s)^{8 / 3} d s\right. \\
& \left.+\int_{T}^{\infty} \int_{s}^{\infty} k(t-s) d t g(s)^{8 / 3} d s\right) .
\end{aligned}
$$

Hence we observe that

$$
\lim _{T \rightarrow \infty} \sup _{j=1,2,3, \ldots} \int_{T}^{\infty}\left(\left\|\int_{0}^{t} K(t-s)\left(\varphi^{2} \psi_{j}(s)\right) d s\right\|_{L_{6}}\right)^{3} d t=0,
$$

provided that

$$
\lim _{T \rightarrow \infty} \sup _{j=1,2,3, \ldots} \int_{T}^{\infty}\left(g_{j}(s)\right)^{8 / 3} d s=0,
$$

where $g_{j}$ denotes $g$ with $\psi$ replaced by $\psi_{j}$.

Set $L_{p}^{T}\left(L_{q}\right):=L_{p}\left([T, \infty) ; L_{q}\right)$. Hölder's inequality implies that

$$
\begin{aligned}
\left(\int_{T}^{\infty} g(s)^{8 / 3} d s\right)^{3 / 8} \leqq & \left(\|\varphi\|_{L_{8}^{T}\left(L_{24 / 7}\right)}\right)^{2} \cdot\|\psi\|_{L_{8}^{T}\left(L_{24 / 7}\right)} \\
& +\left(\|\varphi\|_{L_{\infty}^{T}\left(L_{2}^{1}\right)} \cdot\|\varphi\|_{L_{24}^{T}\left(L_{24 / 5}\right)} \cdot\|\psi\|_{L_{3}^{T}\left(L_{6}\right)}+\cdots\right),
\end{aligned}
$$

where the dots indicate two terms obtained from the first by cyclic permutations of $\varphi, \varphi, \psi$. Moreover, using Sobolev inequalities and interpolation inequalities (see [2] Lemma 4),

$$
\begin{aligned}
& \|v\|_{L_{8}^{T}\left(L_{24 / 7}\right)} \leqq C\left(\|v\|_{\mathrm{Z}}\right)^{1-\theta_{1}} \cdot\left(\|v\|_{L_{3}^{T}\left(L_{6}\right)}\right)^{\theta_{1}} \\
& \|v\|_{L_{24}^{T}\left(L_{24 / 5}\right)} \leqq C\left(\|v\|_{\mathrm{Z}}\right)^{1-\theta_{2}} \cdot\left(\|v\|_{L_{3}^{T}\left(L_{6}\right)}\right)^{\theta_{2}}
\end{aligned}
$$

for some $\theta_{1}, \theta_{2}>0$. Thus we conclude that $\int_{T}^{\infty} g_{j}(s)^{8 / 3} d s$ can be made arbitrary small, uniformly in $\left\{\psi_{j}\right\}$, choosing $T$ large, since $\|\varphi\|_{L_{3}^{T}\left(L_{6}\right)}$ can be arbitrary small. This proves (9). 


$$
\lim _{T \rightarrow \infty} \sup _{j=1,2,3, \ldots} \int_{-\infty}^{-T}\left(\left\|\int_{0}^{t} K(t-s)\left(\varphi^{2} \psi_{j}(s)\right) d s\right\|_{L_{6}}\right)^{3} d t=0
$$

is proven in the same way.

\section{Step 2.}

Choose $\chi \in C_{0}^{\infty}\left(\mathbf{R}^{3}\right)$ such that $\chi=1$ in $B_{1}(0)$, supp $\chi \subset B_{2}(0)$ and $0 \leqq \chi \leqq 1$. Here $B_{\rho}(y)$ denotes the set $\left\{x \in \mathbf{R}^{3}:|x-y| \leqq \rho\right\}$. Moreover set $\chi_{R}(x):=\chi\left(\frac{x}{R}\right), \eta_{R}:=1-\chi_{R}$ and $L_{T_{\infty}}\left(L_{6}\right):=L_{\infty}\left([-T, T] ; L_{6}\right)$. We claim that, for all $T>0$,

$$
\lim _{R \rightarrow \infty} \sup _{j=1,2,3, \ldots}\left\|\int_{0}^{t} K(t-s)\left(\varphi^{2} \eta_{R} \psi_{j}(s)\right) d s\right\|_{L_{T \infty}\left(L_{6}\right)}=0 .
$$

For this it is enough to prove

$$
\underset{R \rightarrow \infty}{\limsup } \sup _{j=1,2,3, \ldots}\left\|\varphi \eta_{R}\right\|_{L_{T \infty}\left(L_{2}^{1}\right)}=0
$$

since

$$
\left\|\int_{0}^{t} K(t-s)\left(\varphi^{2} \eta_{R} \psi(s)\right) d s\right\|_{L_{T \infty}\left(L_{2}^{1}\right)} \leqq C\left\|\varphi \eta_{R}\right\|_{L_{T \infty}\left(L_{2}^{1}\right)},
$$

where $C$ only depends on $\|\varphi\|_{\mathbf{Z}}$ and $\|\psi\|_{\mathbf{Z}}$. We recall that $\varphi=\varphi(u)$ corresponds to data $u=\left(u_{1}, u_{2}\right) \in L_{2}^{1} \oplus L_{2}$, which yields $\left\|\eta_{R} u\right\|_{\mathbf{X}} \rightarrow 0$ as $R \rightarrow \infty$. Say $\left\|\eta_{R_{0}} u\right\|_{\mathbf{X}}<\varepsilon$. This implies that $\left\|\varphi \eta_{R}\right\|_{L_{T \infty}\left(L_{2}^{1}\right)}<C \varepsilon$ for $R>2 R_{0}+T$, where $C$ only depends on $\eta$, as a consequence of hyperbolicity (the finite speed of propagation $=1$ ) and uniqueness of solution of (1) (see e.g. [4]), giving

$$
\varphi(u)(t, x)=\varphi\left(\eta_{R_{0}} u\right)(t, x)
$$

for $t \in[-T, T]$ and $|x|>2 R_{0}+T$, and the energy estimate

$$
\|(\varphi(\bar{u}) \dot{\varphi}(\bar{u}))\|_{L_{\infty}(\mathbf{R} ; \mathbf{X})} \leqq C\|\bar{u}\|_{\mathbf{X}}
$$

for all $\bar{u} \in \mathbf{X}$.

\section{Step 3.}

The purpose of Step 1 and Step 2 was to localize $t$ and $x$ to bounded sets, namely a bounded interval and a ball respectively. We can now apply the Arzela-Ascoli theorem.

Lemma 3.3. A sequence $\left\{f_{j}\right\}_{j=0}^{\infty}$ in $C\left([-T, T] ; L_{6}\left(B_{R}(0)\right)\right.$ has a convergent subsequence iff $\left\{f_{j}\right\}_{j=0}^{\infty}$ is equicontinuous on $[-T, T]$ and, for each $t \in[-T, T]$, the sequence $\left\{f_{j}(t)\right\}_{j=0}^{\infty}$ has a convergent subsequence in $L_{6}\left(B_{R}(0)\right)$.

To prove that

$$
\left\{\int_{0}^{t} K(t-s)\left(\varphi^{2} \chi_{R} \psi_{j}(s)\right) d s\right\} \subset C\left([-T, T] ; L_{6}\right)
$$


and the equicontinuity on $[-T, T]$ we argue as follows:

$$
\begin{aligned}
& \left\|\int_{0}^{t+h} K(t+h-s)\left(\varphi^{2} \chi_{R} \psi(s)\right) d s-\int_{0}^{t} K(t-s)\left(\varphi^{2} \chi_{R} \psi(s)\right) d s\right\|_{L_{6}} \\
& \leqq\left\|\int_{0}^{t}(K(t+h-s)-K(t-s))\left(\varphi^{2} \chi_{R} \psi(s)\right) d s\right\|_{L_{6}} \\
& +\left\|\int_{t}^{t+h} K(t+h-s)\left(\varphi^{2} \chi_{R} \psi(s)\right) d s\right\|_{L_{6}} \\
& \leqq\left(\left\|\int_{0}^{t}(K(t+h-s)-K(t-s))\left(\varphi^{2} \chi_{R} \psi(s)\right) d s\right\|_{L_{2}}\right)^{1 / 9} \\
& \cdot\left(\left\|\int_{0}^{t}(K(t+h-s)-K(t-s))\left(\varphi^{2} \chi_{R} \psi(s)\right) d s\right\|_{L_{8}}\right)^{8 / 9} \\
& +\left\|\int_{t}^{t+h} K(t+h-s)\left(\varphi^{2} \chi_{R} \psi(s)\right) d s\right\|_{L_{6}} .
\end{aligned}
$$

Set $K_{0}(t):=\cos \left(t \sqrt{-\Delta+m^{2}}\right)$. This implies that

$$
\begin{aligned}
& \left\|\int_{0}^{t}(K(t+h-s)-K(t-s))\left(\varphi^{2} \chi_{R} \psi(s)\right) d s\right\|_{L_{2}} \\
& \quad=|h|\left\|\int_{0}^{t} \int_{0}^{1} K_{0}(t+\theta h-s)\left(\varphi^{2} \chi_{R} \psi(s)\right) d \theta d s\right\|_{L_{2}} \\
& \quad \leqq|h| \int_{0}^{t} \int_{0}^{1}\left\|\varphi^{2} \chi_{R} \psi(s)\right\|_{L_{2}} d \theta d s \\
& \left.\quad \leqq|h| \cdot C\|\varphi\|_{\mathbf{z}}\|\psi\|_{\mathbf{z}}\left\|\varphi \chi_{R}\right\|_{L_{T \infty}\left(L_{2}\right)}\right) \quad t \in[-T, T] .
\end{aligned}
$$

Here we have applied the trivial estimate

$$
\left\|K_{0}(t) g\right\|_{L_{2}} \leqq C\|g\|_{L_{2}}, \quad t \in \mathbf{R} .
$$

Furthermore we get

$$
\begin{aligned}
& \left\|\int_{0}^{t}(K(t+h-s)-K(t-s))\left(\varphi^{2} \chi_{R} \psi(s)\right) d s\right\|_{L_{8}} \\
& \quad \leqq \int_{0}^{t}\left\|K(t+h-s)\left(\varphi^{2} \chi_{R} \psi(s)\right)\right\|_{L_{8}} d s+\int_{0}^{t}\left\|K(t-s)\left(\varphi^{2} \chi_{R} \psi(s)\right)\right\|_{L_{8}} d s \\
& \quad \leqq C\|\varphi\|_{\mathbf{Z}}\|\psi\|_{\mathbf{Z}}\left\|\varphi \chi_{R}\right\|_{L_{T \infty}\left(L_{2}^{1}\right)}, \quad t \in[-T, T] .
\end{aligned}
$$

Finally

$$
\left\|\int_{t}^{t+h} K(t+h-s)\left(\varphi^{2} \chi_{R} \psi(s)\right) d s\right\|_{L_{6}} \leqq \int_{t}^{t+h}\left\|K(t+h-s)\left(\varphi^{2} \chi_{R} \psi(s)\right)\right\|_{L_{6}} d s
$$

is treated, as in Step 1 applying $\int_{t}^{t+h} k(t+h-s) d s \rightarrow 0$ as $h \downarrow 0$, uniformly in $t \in \mathbf{R}$, to 
yield

$$
\lim _{h \downarrow 0} \sup _{j=1,2,3, \ldots .}\left\|\int_{t}^{t+h} K(t+h-s)\left(\varphi^{2} \chi_{R} \psi_{j}(s)\right) d s\right\|_{L_{6}}=0
$$

uniformly in $t \in[-T, T]$. This proves the equicontinuity.

It remains to show that, for all $t \in[-T, T]$,

$$
\mathscr{K}(t):=\left\{\int_{0}^{t} K(t-s)\left(\varphi^{2} \chi_{R} \psi_{j}(s)\right) d s: j=1,2,3, \ldots\right\}
$$

has a convergent subsequence in $L_{6}$. So, fix a $t \in[-T, T]$ and $\delta>0$ small enough, to be determined later, and let $\chi_{[0, t]}$ denote the characteristic function for the interval $[0, t]$, if $t>0$, (and $\chi_{[t, 0]}$ the characteristic function for the interval $[t, 0]$, if $t<0)$. Moreover, let $F^{\kappa}, \kappa \in \mathbf{R}$, denote the operators on real $L_{2}([-T, T])$-functions $h$ defined by $\left(F^{\kappa} h\right)_{n}^{\wedge}=(i n)^{\kappa} \hat{h}_{n}, n \in \mathbf{Z}-\{0\}$ and $\left(F^{\kappa} h\right)_{0}^{\wedge}=\hat{h}_{0}$, where ${ }^{\wedge}$ denotes the Fourier operator. For $t>0$, we then obtain with Parseval's formula

$$
\begin{aligned}
& \left\|\int_{0}^{t} K(t-s)\left(\varphi^{2} \chi_{R} \psi(s)\right) d s\right\|_{L_{2}^{1+\delta}} \\
& \quad \leqq\left\|\int_{-T}^{T}\left(F^{\kappa} \chi_{[0, t]}\right)(s) \cdot\left(F^{-\kappa} K(t-\cdot)\left(\varphi^{2} \chi_{R} \psi(\cdot)\right)\right)(s) d s\right\|_{L_{2}^{1+\delta}} \\
& \quad \leqq\left\|F^{\kappa} \chi_{[0, t]}\right\|_{L_{2}([-T, T])} \cdot\left\|F^{-\kappa} K(t-\cdot)\left(\varphi^{2} \chi_{R} \psi(\cdot)\right)\right\|_{L_{2}\left([-T, T] ; L_{2}^{1+\delta}\right)} .
\end{aligned}
$$

Here we note that, for $\kappa<1 / 2$, we have $\left\|F^{\kappa} \chi_{[0, t]}\right\|_{L_{2}([-T, T])}<\infty$. This is trivial since $\left\|F^{\kappa} \chi_{[0, t]}\right\|_{L_{2}([-T, T])} \leqq C\left(\sum_{n \in \mathbf{Z}-\{0\}}\left(n^{\kappa} \cdot \frac{1}{n}\right)^{2}+1\right)^{1 / 2}$. The second factor is treated by the following lemma due to Bernner [6]

Lemma 3.4. For $0 \leqq \kappa<1$ and $s \geqq 1$, then

This yields

$$
\left\|F^{-\kappa} K(\cdot) f(\cdot)\right\|_{L_{2}\left([-T, T] ; L_{2}^{s+\wedge}\right)} \leqq C\|f(\cdot)\|_{L_{2}\left([-T, T] ; L_{2}^{s-1}\right)} \cdot
$$

$$
\left\|F^{-\kappa} K(t-\cdot)\left(\varphi^{2} \chi_{R} \psi(\cdot)\right)\right\|_{L_{2}\left([-T, T] ; L_{2}^{1+\delta}\right)} \leqq C\left\|\varphi^{2} \chi_{R} \psi\right\|_{L_{2}\left([-T, T] ; L_{2}^{\delta-\kappa}\right)} \cdot
$$

Pick $\delta=\kappa<1 / 2$ and observe that

$$
\left\|\varphi^{2} \chi_{R} \psi\right\|_{L_{2}\left([-T, T] ; L_{2}\right)} \leqq C\left(\|\varphi\|_{\mathrm{Z}}\right)^{2}\|\psi\|_{\mathrm{Z}}
$$

where $C$ depends on $T$ and $R$. The compactness of $\mathscr{K}(t)$ follows from the RellichKondrachov theorem. The case $t<0$ is treated similarity.

\section{Step 4.}

For each $N \in\{1,2,3, \ldots\}$ choose a $T(N)($ Step 1) and then a $R(N)($ Step 2) such that

and

$$
\sup _{j=1,2,3, \ldots}\left\|T_{u} \psi_{j}\right\|_{L_{3}\left((-\infty,-T(N)] \cup[T(N), \infty) ; L_{6}\right)}<\frac{1}{N}
$$

$$
\sup _{j=1,2,3, \ldots}\left\|T_{u}^{(R(N))} \psi_{j}\right\|_{L_{3}\left([-T(N), T(N)] ; L_{6}\right)}<\frac{1}{N},
$$


where $T_{u}^{(R(N))} \psi(t):=\int_{0}^{t} K(t-s)\left(\varphi^{2} \eta_{R(N)} \psi(s)\right) d s$. Then choose inductively subsequences $\left\{\psi_{j, N}\right\}$ of $\left\{\psi_{j, N-1}\right\}, N=1,2, \ldots$, with $\psi_{j, 0}=\psi_{j}$, such that $\left\{T_{u, R(N)} \psi_{j, N}\right\}$ converges in $L_{3}\left([-T(N), T(N)] ; L_{6}\right)$, where $T_{u, R(N)}:=T_{u}-T_{u}^{(R(N))}($ Step 3$)$. Then the subsequence $\left\{\psi_{N, N}\right\}_{N=1}^{\infty}$ converges in $L_{3}\left(L_{6}\right)$. This completes the proof of Step 4 and Theorem 1.2.

\section{Proof of Theorem 1.1}

In [2] Baez and Zhou prove that $W_{ \pm}$and $S$ are analytic mappings from a neighborhood of $\mathbf{X}$ to $\mathbf{X}$. They also claim that if one could show that $u \mapsto \varphi(u)$ is analytic from $\mathbf{X}$ to $\mathbf{Z}$ then the analyticity of $W_{ \pm}$and $S$ would follow. It is not clear to us how their argument should be generalized to give this result. Instead we supply a new proof based on the following fact, see e.g. [8].

Lemma 4.1. Let $A_{k}: \mathbf{X} \rightarrow \mathbf{X}, k=1,2, \ldots$ be analytic mappings, uniformly bounded on all compact sets $D \subset \mathbf{X}$. Also assume that $A_{k} u \rightarrow A u$ as $k \rightarrow \infty$ for all $u \in \mathbf{X}$. Then the mapping $A: \mathbf{X} \rightarrow \mathbf{X}$ is analytic.

We claim that it is enough to prove the following lemma.

Lemma 4.2. For all compact sets $D \subset \mathbf{X}$,

$$
\sup _{u \in D}\|\varphi\|_{L_{3}\left(L_{6}\right)}<\infty
$$

To see this let $U\left(T_{2}, T_{1}\right): \mathbf{X} \rightarrow \mathbf{X}$ denote the mapping $\left.u \mapsto(\varphi(u), \dot{\varphi}(u))\right|_{t=T_{2}}$, where $\varphi(u)$ is the solution of (1) with data $\left.(\varphi, \dot{\varphi})\right|_{t=T_{1}}=u$. Note that $U\left(T_{2}, T_{1}\right) u \in \mathbf{X}$ follows from conservation of energy. Moreover the wave operators and their inverses can be defined as

$$
W_{ \pm}=\lim _{T \rightarrow \pm \infty} U(-T, 0) U_{0}(T)
$$

and

$$
W_{ \pm}^{-1}=\lim _{T \rightarrow \pm \infty} U_{0}(-T) U(T, 0) .
$$

However all operators $U_{0}\left(T_{3}\right) U\left(T_{2}, T_{1}\right)$ and $U\left(T_{3}, T_{2}\right) U_{0}\left(T_{1}\right), T_{1}, T_{2}, T_{3} \in \mathbf{R}$, are analytic since the mapping $u \mapsto \varphi(u)$ is analytic, linear mappings are analytic and analytic mappings are closed under composition. Also, $U_{0}(T)$ being an isometry on $\mathbf{X}$, Lemma 4.1 implies that $W_{ \pm}, W_{ \pm}^{-1}$ and $S$ are analytic provided

$$
\sup _{u \in D} \sup _{T_{1}, T_{2} \in \mathbf{R}}\left\|U\left(T_{2}, T_{1}\right) u\right\|_{\mathbf{X}}<\infty
$$

for all compact sets $D \subset \mathbf{X}$. $U\left(T_{2}, T_{1}\right)$ only depends on $T_{2}-T_{1}$ so it is enough to consider $T_{2}=T$ and $T_{1}=0$. Then $U(T, 0)=U(T)$ and

$$
U(T) u=U_{0}(T) u+\int_{0}^{T} U_{0}(T-s) N(U(s) u) d s
$$


with notation from Sect. 1. This yields

$$
\begin{aligned}
\|U(T) u\|_{\mathbf{x}} & \leqq\left\|U_{0}(T) u\right\|_{\mathbf{x}}+\left|\int_{0}^{T}\left\|U_{0}(T-s) N(U(s) u)\right\|_{\mathbf{X}} d s\right| \\
& =\|u\|_{\mathbf{X}}+\lambda\left|\int_{0}^{T}\left(\|\varphi(u)(s)\|_{L_{6}}\right)^{3} d s\right|, \quad T \in \mathbf{R} .
\end{aligned}
$$

Hence it is enough to prove

$$
\sup _{u \in D}\|\varphi(u)\|_{L_{3}\left(L_{6}\right)}<\infty .
$$

Proof of Lemma 4.2. To prove that $\|\varphi(u)\|_{\mathrm{X}}$ is uniformly bounded for $u$ in compact subsets of $\mathbf{X}$, we follow the method in [4] that goes back to Morawetz and Strauss. For the convenience of the reader we give a complete proof. Throughout this section we let $C$ denote constants that only depend on numerical entities, $\lambda, \eta, p$ and others, that are independent of $u$, while $C(D)$ denotes constants that also depends boundedly on $\|u\|_{\mathbf{x}}$. The value of the different constants $C(D)$ may vary from line to line. Only occasionally we index the constants for reference. Moreover, for simplicity, we write $\varphi$ and $\varphi_{0}$ for $\varphi(u)$ and $\varphi_{0}(u)$ respectively.

The proof is carried out through a sequence of five lemmas. We start with an application of Hölder's inequality and the Sobolev embeddings.

Lemma 4.3. There exists a constant $C$, such that for all $\eta \in\left[-\frac{1}{2}, 1\right]$,

$$
\begin{aligned}
\left\|\varphi^{3}\right\|_{L_{8 / 7}^{1}} & \leqq C\left(\|\varphi\|_{L_{2}^{1}}\right)^{2+\eta}\left(\|\varphi\|_{L_{8}}\right)^{1-\eta}, \\
\left\|\varphi^{3}\right\|_{L_{10 / 9}^{1}} & \leqq C\left(\|\varphi\|_{L_{2}^{1}}\right)^{2+\eta}\left(\|\varphi\|_{L_{10}}\right)^{1-\eta} .
\end{aligned}
$$

Next we show that (10) follows from

$$
\sup _{u \in D}\|\varphi\|_{L_{4}\left(L_{8}\right)}<\infty .
$$

Proof of (11) implies (10): Hölder's inequality yields

$$
\left\|\int_{0}^{t} K(t-s) \varphi^{3}(s) d s\right\|_{L_{6}} \leqq\left(\left\|\int_{0}^{t} K(t-s) \varphi^{3}(s) d s\right\|_{L_{2}}\right)^{1 / 9}\left(\left\|\int_{0}^{t} K(t-s) \varphi^{3}(s) d s\right\|_{L_{8}}\right)^{8 / 9}
$$

Hence by (8) and by conservation of energy

$$
\left\|\int_{0}^{t} K(t-s) \varphi^{3}(s) d s\right\|_{L_{2}} \leqq C\left(\left\|\varphi_{0}\right\|_{L_{\infty}\left(L_{2}^{1}\right)}+\|\varphi\|_{L_{\infty}\left(L_{2}^{1}\right)}\right) \leqq C(D) .
$$

The second factor is estimated by Proposition 3.1

$$
\left\|\int_{0}^{t} K(t-s) \varphi^{3}(s) d s\right\|_{L_{8}} \leqq C\left|\int_{0}^{t} k(t-s)\left\|\varphi^{3}(s)\right\|_{L_{8 / 7}^{1}} d s\right|,
$$

where $k(t) \in L_{1}(\mathbf{R})$. Moreover by Lemma 4.3

$$
\left\|\varphi^{3}(s)\right\|_{L_{8 / 7}^{1}} \leqq C\left(\|\varphi(s)\|_{L_{2}^{1}}\right)^{3 / 2}\left(\|\varphi(s)\|_{L_{8}}\right)^{3 / 2},
$$


so again by conservation of energy

$$
\left\|\int_{0}^{t} K(t-s) \varphi^{3}(s) d s\right\|_{L_{8}} \leqq C(D)\left|\int_{0}^{t} k(t-s)\left(\|\varphi(s)\|_{L_{8}}\right)^{3 / 2} d s\right| .
$$

Finally Young's inequality, Lemma 3.2 and (8) imply

$$
\sup _{u \in D}\|\varphi\|_{L_{3}\left(L_{6}\right)} \leqq C(D)\left(1+\sup _{u \in D}\|\varphi\|_{L_{4}\left(L_{8}\right)}\right)^{4 / 3} .
$$

This completes the proof of (11) implies (10).

Next we state a lemma from [4].

Lemma 4.4. For $q \in[4,12]$,

$$
\left\|\varphi_{0}\right\|_{L_{4}\left(L_{q}\right)} \leqq C(D) .
$$

For a proof of this space-time estimate we refer to [5].

Lemma 4.5. Let $k(t)$ be given by Proposition 3.1. Then

$$
\sup _{t \in \mathbf{R}}\left|\int_{0}^{t} k(t-s)\|\varphi(s)\|_{L_{8}} d s\right| \leqq C(D)
$$

and

$$
\sup _{t \in \mathbf{R}}\left|\int_{0}^{t} k(t-s)\|\varphi(s)\|_{L_{10}} d s\right| \leqq C(D)
$$

Proof of Lemma 4.5. Proposition 3.1, Lemma 4.3 and conservation of energy applied to (8) give

$$
\begin{aligned}
\|\varphi(t)\|_{L_{8}} & \leqq\left\|\varphi_{0}(t)\right\|_{L_{8}}+\int_{0}^{t} k(t-s)\left\|\varphi^{3}(s)\right\|_{L_{8 / 7}^{1}} d s \\
& \leqq\left\|\varphi_{0}(t)\right\|_{L_{8}}+C(D) \int_{0}^{t} k(t-s)\left(\|\varphi(s)\|_{L_{8}}\right)^{1-\eta} d s
\end{aligned}
$$

for some $\eta \in(0,1)$. Multiply by $k(\sigma-t)$ and integrate over $(0, \sigma)$. We obtain

$$
\begin{aligned}
\int_{0}^{\sigma} k(\sigma-t)\|\varphi(t)\|_{L_{8}} d t \leqq & \int_{0}^{\sigma} k(\sigma-t)\left\|\varphi_{0}(t)\right\|_{L_{8}} d t \\
& +C(D) \int_{0}^{\sigma} k(\sigma-t) \int_{0}^{t} k(t-s)\left(\|\varphi(s)\|_{L_{8}}\right)^{1-\eta} d s d t .
\end{aligned}
$$

Here we use

$$
\begin{aligned}
& \int_{0}^{\sigma} k(\sigma-t) \int_{0}^{t} k(t-s)\left(\|\varphi(s)\|_{L_{8}}\right)^{1-\eta} d s d t \\
& \quad \leqq \int_{0}^{\sigma} k(\sigma-t)\left(\int_{0}^{t} k(t-s)\|\varphi(s)\|_{L_{8}} d s\right)^{1-\eta}\left(\int_{0}^{t} k(s) d s\right)^{\eta} d t
\end{aligned}
$$


together with the notation

$$
\begin{aligned}
g(t) & :=\int_{0}^{t} k(t-s)\|\varphi(s)\|_{L_{8}} d s \\
g_{0}(t): & =\int_{0}^{t} k(t-s)\left\|\varphi_{0}(s)\right\|_{L_{8}} d s
\end{aligned}
$$

to obtain

$$
g(\sigma) \leqq g_{0}(\sigma)+C(D) \sup _{t \leqq \sigma} g(t)^{1-\eta}
$$

But $k \in L_{4 / 3} \cap L_{1}$ so Lemma 4.4 gives

$$
\sup _{\sigma \in \mathbf{R}} g_{0}(\sigma) \leqq C(D)
$$

and hence (12) implies

$$
\sup _{\sigma \in \mathbf{R}} g(\sigma) \leqq C(D) .
$$

This proves the first part of the statement. The argument for the other part is similar.

Next lemma gives a weak decay estimate that is crucial.

\section{Lemma 4.6.}

$$
\lim _{t^{*} \rightarrow \infty} \sup _{t \geqq t^{*}} \int_{t^{*}}^{t} k(t-s)\|\varphi(s)\|_{L_{8}} d s=0
$$

and

$$
\lim _{t^{*} \rightarrow \infty} \sup _{t \geqq t^{*}} \int_{-t}^{-t^{*}} k(t-s)\|\varphi(s)\|_{L_{8}} d s=0
$$

uniformly for $u \in D$.

Proof of Lemma 4.6. We start by proving the result for the case with compactly supported data. Moreover we first prove the following claim:

For $\varepsilon, T$ and $S$ positive numbers there exists a $S_{1}>S$, independent of the support of data, and an interval $I=[\hat{t}-T, \hat{t}] \subset\left[S, S_{1}\right]$ such that for $t \leqq \hat{t}$,

$$
\int_{\hat{t}-T}^{t} k(t-s)\|\varphi(s)\|_{L_{8}} d s<\varepsilon .
$$

To prove this we apply a famous lemma of Morawetz and Strauss.

Lemma 4.7. Assume that data $u \in \mathbf{X}$ has support contained in $|x| \leqq R_{0}<\infty$. Let $\varepsilon_{0}, T, S$ be positive numbers. Then there exists an $S_{1}$ depending boundedly on $S, T, \varepsilon_{0}$ and $\|u\|_{\mathbf{X}}$ but not $R_{0}$, and there exists an interval $I=[\hat{t}-2 T, \hat{t}] \subseteq\left[S, S_{1}\right]$ such that

$$
\iint_{I}|\varphi(x, t)|^{4} d x d t<\varepsilon_{0}
$$

For a proof we refer to [11]. 


\section{Multiply}

$$
\|\varphi(\tau)\|_{L_{8}} \leqq\left(\|\varphi(\tau)\|_{L_{4}}\right)^{1 / 6}\left(\|\varphi(\tau)\|_{L_{10}}\right)^{5 / 6}
$$

by $k(s-\tau)$ and integrate over $(s-T, s), s>T$. Hölder's inequality and Lemma 4.5 yield

$$
\int_{s-T}^{s} k(s-\tau)\|\varphi(\tau)\|_{L_{8}} d \tau \leqq C(D)\left(\int_{s-T}^{s} \int|\varphi(x, \tau)|^{4} d x d \tau\right)^{1 / 24}
$$

Moreover, Proposition 3.1 and (8) give

$$
\|\varphi(t)\|_{L_{8}} \leqq\left\|\varphi_{0}(t)\right\|_{L_{8}}+C(D)\left(\int_{0}^{t-T}+\int_{t-T}^{t} k(t-s)\left(\|\varphi(s)\|_{L_{8}}\right)^{1-\eta} d s\right)
$$

for some $\eta \in(0,1)$ and so

$$
\begin{aligned}
\int_{\hat{t}-T}^{t} k(t-s)\|\varphi(s)\|_{L_{8}} d s \leqq & \int_{\hat{t}-T}^{t} k(t-s)\left\|\varphi_{0}(s)\right\|_{L_{8}} d s \\
& +C(D)\left(\int_{\hat{t}-T}^{t} k(t-s) \int_{0}^{s-T} k(s-\tau)\left(\|\varphi(\tau)\|_{L_{8}}\right)^{1-\eta} d \tau d s\right. \\
& \left.+\int_{\hat{t}-T}^{t} k(t-s) \int_{s-T}^{s} k(s-\tau)\left(\|\varphi(\tau)\|_{L_{8}}\right)^{1-\eta} d \tau d s\right)
\end{aligned}
$$

Here by Lemma 4.5

$$
\begin{aligned}
& \left(\int_{\hat{t}-T}^{t} k(t-s) \int_{0}^{s-T} k(s-\tau)\left(\|\varphi(\tau)\|_{L_{8}}\right)^{1-\eta} d \tau d s\right. \\
& \quad \leqq \int_{\hat{t}-T}^{t} k(t-s)\left(\int_{T}^{s} k(\tau) d \tau\right)^{\eta}\left(\int_{0}^{s-T} k(s-\tau)\|\varphi(\tau)\|_{L_{8}} d \tau\right)^{1-\eta} d s \\
& \quad \leqq C(D) \int_{\hat{t}-T}^{t} k(t-s)\left(\int_{T}^{t} k(\tau) d \tau\right)^{\eta} d s \leqq C(D) T^{\eta(1-(2+\theta) 3 / 8)} \rightarrow 0
\end{aligned}
$$

as $T \rightarrow \infty$, for $(2+\theta) 3 / 8>1$ (e.g. $\theta=1)$, and

$$
\begin{aligned}
& \left(\int_{\hat{t}-T}^{t} k(t-s) \int_{s-T}^{s} k(s-\tau)\left(\|\varphi(\tau)\|_{L_{8}}\right)^{1-\eta} d \tau d s\right. \\
& \quad \leqq \int_{\hat{t}-T}^{t} k(t-s)\left(\int_{0}^{T} k(\tau) d \tau\right)^{\eta}\left(\int_{s-T}^{s} k(s-\tau)\|\varphi(\tau)\|_{L_{8}} d \tau\right)^{1-\eta} d s \\
& \quad \leqq C(D) \int_{\hat{t}-T}^{t} k(t-s)\left(\int_{s-T}^{s} \int|\varphi(x, \tau)|^{4} d x d \tau\right)^{(1-\eta) / 24} d s \\
& \quad \leqq C(D) \int_{\hat{t}-T}^{t} k(t-s)\left(\int_{\hat{t}-2 T}^{t} \int|\varphi(x, \tau)|^{4} d x d \tau\right)^{(1-\eta) / 24} d s \\
& \quad \leqq C(D)\left(\int_{\hat{t}-2 T}^{t} \int|\varphi(x, \tau)|^{4} d x d \tau\right)^{(1-\eta) / 24} .
\end{aligned}
$$


Now let $T$ be sufficiently large and let $\hat{t} \geqq 2 T+S$ in Lemma 4.7. Then for $t \leqq \hat{t}$ we get

$$
\int_{\hat{t}-T}^{t} k(t-s)\|\varphi(s)\|_{L_{8}} d s \leqq C(D)\left(\varepsilon_{0}+\varepsilon_{0}^{(1-\eta) / 24}\right)<\varepsilon
$$

for $\varepsilon_{0}$ sufficiently small. The claim is proved.

To prove the statement of Lemma 4.6, still with the additional assumption on the support of data, we have to show that given an $\varepsilon>0$, that can be assumed to be sufficiently small, there exists a $t^{*}$ such that

$$
\sup _{u \in D} \sup _{t \geqq t^{*}}\left|\int_{t^{*}}^{t} k(t-s)\|\varphi(s)\|_{L_{8}} d s\right| \leqq \varepsilon .
$$

This will technically be done by first choosing $\eta>0$ sufficiently small (and $\varepsilon$ sufficiently small with reference to this choice of $\eta$ ) and then $T$ sufficiently large and finally $t^{*}$ sufficiently large and through this procedure show that

$$
\int_{t^{*}-T}^{t} k(t-s)\|\varphi(s)\|_{L_{8}} d s \leqq \varepsilon
$$

for all $u \in D$ and $t \geqq t^{*}-T$.

With reference to Proposition 3.1, let $\eta>0$ be so small such that $k \in L_{1} \cap L_{4 /(3-\eta)}$ for some choice of $\theta \in(0,1]$, where $p^{\prime}=8$.

Let $t^{*}-T$ be so large such that

$$
\int_{t^{*}-T}^{t} k(t-s)\left\|\varphi_{0}(s)\right\|_{L_{8}} d s<\frac{1}{3} \varepsilon, \quad t^{*} \leqq t,
$$

and

$$
\int_{t^{*}-T}^{t} k(t-s)\left(\left\|\varphi_{0}(s)\right\|_{L_{8}}\right)^{1+\eta} d s<\frac{1}{3} \varepsilon^{1+\eta}, \quad t^{*} \leqq t
$$

This can be done since $\varphi_{0} \in L_{4}\left(L_{8}\right)$ :

$$
\int_{t^{*}-T}^{t} k(t-s)\left\|\varphi_{0}(s)\right\|_{L_{8}} d s \leqq\left(\int_{0}^{t+T-t^{*}} k(s)^{4 / 3} d s\right)^{3 / 4}\left(\int_{t^{*}-T}^{t}\left(\left\|\varphi_{0}(s)\right\|_{L_{8}}\right)^{4} d s\right)^{1 / 4}
$$

and

$$
\begin{aligned}
& \int_{t^{*}-T}^{t} k(t-s)\left(\left\|\varphi_{0}(s)\right\|_{L_{8}}\right)^{1+\eta} d s \\
& \quad \leqq\left(\int_{0}^{t+T-t^{*}} k(s)^{4 /(3-\eta)} d s\right)^{(3-\eta) / 4}\left(\int_{t^{*}-T}^{t}\left(\left\|\varphi_{0}(s)\right\|_{L_{8}}\right)^{4} d s\right)^{(1+\eta) / 4}
\end{aligned}
$$

Set

$$
C_{1}:=\left(\int_{0}^{\infty} k(s)^{4 / 3} d s\right)^{3 / 4}+\left(\int_{0}^{\infty} k(s)^{4 /(3-\eta)} d s\right)^{(3-n) / 4}+1
$$


and choose $t^{*}-T$ such that

$$
\left(\int_{t^{\star}-T}^{\infty}\left(\left\|\varphi_{0}(s)\right\|_{L_{8}}\right)^{4} d s\right)^{3 / 4} \leqq \varepsilon / 3 C_{1} .
$$

Next we show that for $\varepsilon$ small enough, only depending on $D$, viz.

$$
\varepsilon \leqq \min \left(\left(2 C_{2}\right)^{-2 / \eta},\left(6 C_{6}\right)^{-2}\right),
$$

where $C_{2}$ and $C_{6}$ are defined in (17) and (20) below, and $T$ large enough, also depending only on $D$, viz.

$$
T \geqq \max \left(\left(\frac{2 C_{3}}{\varepsilon^{1+\eta / 2}}\right)^{1 /((2+\theta) 3 / 8-1)},\left(\frac{6 C_{4}}{\varepsilon}\right)^{1 /((2+\theta) 3 / 8-1)},\left(\frac{6 C_{5}}{\varepsilon}\right)^{1 / \varepsilon_{2}}\right),
$$

where $C_{3}, C_{4}$ and $C_{5}$ are defined in (17), (18) and (19) below,

$$
\int_{t^{*}-T}^{t} k(t-s)\left(\|\varphi(s)\|_{L_{8}}\right)^{1+\eta} d s \leqq \varepsilon^{1+\eta / 2}, \quad t \geqq t^{*},
$$

provided

$$
\int_{t^{*}-T}^{t} k(\tau-s)\|\varphi(s)\|_{L_{8}} d s \leqq \varepsilon
$$

for all $\tau \in\left[t^{*}-T, t\right]$. To prove this we apply Lemma 4.3 to (8) to obtain

$$
\left(\|\varphi(s)\|_{L_{8}}\right)^{1+\eta} \leqq 2^{\eta}\left(\left(\left\|\varphi_{0}(s)\right\|_{L_{8}}\right)^{1+\eta}+C(D)\left(\int_{0}^{s} k(s-\sigma)\|\varphi(\sigma)\|_{L_{8}} d \sigma\right)^{1+\eta}\right),
$$

and so

$$
\sup _{s \in \mathbf{R}} \int_{0}^{s} k(s-\sigma)\left(\|\varphi(\sigma)\|_{L_{8}}\right)^{1+\eta} d \sigma \leqq C(D)
$$

and

$$
\begin{aligned}
\int_{t^{*}-T}^{t} k(t-s)\left(\|\varphi(s)\|_{L_{8}}\right)^{1+\eta} d s & \\
\leqq & 2^{\eta} \int_{t^{*}-T}^{t} k(t-s)\left(\left\|\varphi_{0}(s)\right\|_{L_{8}}\right)^{1+\eta} d s \\
& +2^{\eta} C(D) \int_{t^{*}-T}^{t} k(t-s)\left(\int_{0}^{s-T / 2}+\int_{s-T / 2}^{s} k(s-\sigma)\|\varphi(\sigma)\|_{L_{8}} d \sigma\right)^{1+\eta} d s \\
\leqq & \varepsilon^{1+\eta}+2 C(D)\left\{\left(\int_{T / 2}^{T} k(s) d s\right)\left(\sup _{s \in \mathbf{R}} \int_{0}^{s} k(s-\sigma)\|\varphi(\sigma)\|_{L_{8}} d \sigma\right)^{1+\eta}\right. \\
& +\int_{t^{*}-T / 2}^{t} k(t-s)\left(\int_{s-T / 2}^{s} k(s-\sigma)\|\varphi(\sigma)\|_{L_{8}} d \sigma\right)^{1+\eta} d s \\
& \left.+\int_{t^{*}-T / 2}^{t} k(t-s)\left(\int_{0}^{s-T / 2} k(s-\sigma)\|\varphi(\sigma)\|_{L_{8}} d \sigma\right)^{1+\eta} d s\right\}
\end{aligned}
$$




$$
\begin{aligned}
\leqq & C(D) \varepsilon^{1+\eta}+C(D) T^{1-(2+\theta) 3 / 8}+C(D) \sup _{s \in\left(t^{*}-T / 2, t\right)}\left\{\int_{0}^{s-T / 2} k(s-\sigma)\|\varphi(\sigma)\|_{L_{8}} d \sigma\right\}^{1+\eta} \\
\leqq & C(D) \varepsilon^{1+\eta}+C(D) T^{1-(2+\theta) 3 / 8} \\
& +C(D)\left(\int_{T / 2}^{\infty} k(s) d s\right)^{\eta} \sup _{s \in \mathbf{R}} \int_{0}^{s} k(s-\sigma)\left(\|\varphi(\sigma)\|_{L_{8}}\right)^{1+\eta} d \sigma \\
\leqq & C_{2}(D) \varepsilon^{1+\eta}+C_{3}(D) T^{1-(2+\theta) 3 / 8} \leqq \varepsilon^{1+\eta / 2}
\end{aligned}
$$

where $\theta \in\left(\frac{2}{3}, 1\right]$, e.g. $\theta=1$.

Moreover, use the claim above to determine a $t^{*}=\hat{t}$ such that for $T$ chosen sufficiently large and $\varepsilon$ sufficiently small and $t^{*}-T$ sufficiently large

Define $t^{* *}$ by

$$
\int_{t^{*}-T}^{t} k(t-s)\|\varphi(s)\|_{L_{8}} d s<\varepsilon, \quad t \leqq t^{*}
$$

$$
t^{* *}:=\sup \left\{t: \int_{t^{*}-T}^{\tau} k(\tau-s)\|\varphi(s)\|_{L_{8}} d s<\varepsilon, \text { all } \tau \in\left[t^{*}, t\right]\right\} .
$$

By definition we have $t^{* *} \geqq t^{*}$. If $t^{* *}=\infty$ our lemma is proved. Assume that $t^{* *}<\infty$. Choose $t$ with $t^{* *}<t \leqq t^{* *}+\varepsilon_{1}, \varepsilon_{1}>0$ small, to be chosen later. We have

$$
\begin{aligned}
& \int_{t^{*}-T}^{t} k(t-s)\|\varphi(s)\|_{L_{8}} d s \\
& \leqq \\
& \int_{t^{*}-T}^{t} k(t-s)\left\|\varphi_{0}(s)\right\|_{L_{8}} d s+C \int_{t^{*}-T}^{t} k(t-s) \int_{0}^{s} k(s-\sigma)\left\|\varphi^{3}(\sigma)\right\|_{L_{8 / 7}^{1}} d \sigma d s \\
& \leqq \\
& \int_{t^{*}-T}^{t} k(t-s)\left\|\varphi_{0}(s)\right\|_{L_{8}} d s+C(D) \int_{t^{*}-T}^{t^{*}-T / 2} k(t-s) \int_{0}^{s} k(s-\sigma)\|\varphi(\sigma)\|_{L_{8}} d \sigma d s \\
& \quad+C(D) \int_{t^{*}-T / 2}^{t^{* *}} k(t-s) \int_{0}^{s-T / 2} k(s-\sigma)\|\varphi(\sigma)\|_{L_{8}} d \sigma d s \\
& \quad+C(D) \int_{t^{* *}}^{t} k(t-s) \int_{s-T / 2}^{s} k(s-\sigma)\left(\|\varphi(\sigma)\|_{L_{8}}\right)^{1+\eta} d \sigma d s \\
& \quad+\int_{t^{* *}}^{t} k(t-s) \int_{0}^{s} k(s-\sigma)\|\varphi(\sigma)\|_{L_{8}} d \sigma d s \\
& \equiv I_{1}+\cdots+I_{5} .
\end{aligned}
$$

Here

$$
\begin{aligned}
I_{1} & \leqq 1 / 3 \varepsilon \\
I_{2} & \leqq C_{4}(D) T^{1-(2+\theta) 3 / 8} \leqq 1 / 6 \varepsilon \\
I_{3} & \leqq D(D)\left(\int_{T / 2}^{\infty} k(s) d s\right)^{\eta /(1+\eta)} \sup _{s \in\left(t^{*-}-T, t^{* *}\right)}\left(\int_{0}^{s} k(s-\sigma)\left(\|\varphi(\sigma)\|_{L_{8}}\right)^{1+\eta} d \sigma\right)^{1 /(1+\eta)} \\
& \leqq C_{5}(D) T^{-\varepsilon_{2}} \leqq 1 / 6 \varepsilon
\end{aligned}
$$

for some $\varepsilon_{2}>0$. 


$$
\begin{aligned}
& I_{4} \leqq C_{6}(D) \varepsilon^{1+\eta / 2} \leqq 1 / 6 \varepsilon \\
& I_{5} \leqq C(D) \int_{t^{* *}}^{t} k(t-s) d s \leqq 1 / 6 \varepsilon,
\end{aligned}
$$

provided $\varepsilon_{1}$ small enough. This implies that

$$
\int_{t^{*}-T}^{t} k(t-s)\|\varphi(s)\|_{L_{8}} d s<\varepsilon
$$

and hence yields a contradiction. The first part of Lemma 4.6 is proven for the case with compactly supported data. The second part has the same proof reversing time direction.

It remains to prove the lemma without the assumption on the support of data. Let $\varphi_{v}$ be the solution of (1) with compactly supported data $u_{v}=\left(u_{v, 1}, u_{v, 2}\right)$ approximating $u=\left(u_{1}, u_{2}\right)$ such that $u_{v, i}(x)=u_{i}(x), i=1,2$ for $|x| \leqq R_{v}$, where $R_{v} \rightarrow \infty$ as $v \rightarrow \infty$ and such that $u_{v}$ is uniformly bounded in $\mathbf{X}$ for $v \geqq 0$. Then $\varphi_{0, v}(x, t)=\varphi_{0}(x, t)$ for $|x| \leqq R_{v}-|t|$, and

$$
\left\|\varphi_{0, v}-\varphi_{0}\right\|_{L_{2}^{1}} \leqq\left\|\varphi_{0, v}\right\|_{L_{2}^{1}\left(|x| \geqq R_{v}-|t|\right)}+\left\|\varphi_{0}\right\|_{L_{2}^{1}\left(|x| \geqq R_{v}-|t|\right)} \rightarrow 0
$$

as $v \rightarrow \infty$. We can also assume that $\left\|\varphi_{0, v}\right\|_{L_{8}}$ is uniformly bounded in $L_{4}(\mathbf{R})$. However from now we will only consider $t>0$. The case $t<0$ is analogous. Thus we have

$$
\begin{aligned}
& \left(\int_{t^{*}}^{t}\left(\left\|\varphi_{0, v}(s)-\varphi_{0}(s)\right\|_{L_{8}}\right)^{4} d s\right)^{1 / 4} \\
& \quad \leqq C\left(\int_{t^{*}}^{t}\left(\left(\left\|\varphi_{0, v}(s)\right\|_{L_{8}\left(|x| \geqq R_{v}-s\right)}\right)^{4}+\left(\left\|\varphi_{0}(s)\right\|_{L_{8}\left(|x| \geqq R_{v}-s\right)}\right)^{4}\right) d s\right)^{1 / 4} \rightarrow 0
\end{aligned}
$$

as $v \rightarrow \infty$.

Choose $t^{*}$, independent of $v$, such that

$$
\int_{t^{*}}^{t} k(t-s)\left\|\varphi_{v}(s)\right\|_{L_{8}} d s<\varepsilon, \quad t \geqq t^{*}
$$

which can be done in the proof above since $S_{1}$ in Lemma 4.7 is independent of the support of data. We obtain

$$
\int_{t^{*}}^{t} k(t-s)\|\varphi(s)\|_{L_{8}} d s \leqq \varepsilon+\int_{t^{*}}^{t} k(t-s)\left\|\varphi_{v}(s)-\varphi(s)\right\|_{L_{8}} d s .
$$

Lemma 4.6 will be proved if we can show that for each $t>t^{*}$,

$$
\int_{t^{*}}^{t} k(t-s)\left\|\varphi_{v}(s)-\varphi(s)\right\|_{L_{8}} d s<\varepsilon,
$$

for $v \geqq v_{*}$. We first prove that, for fixed $t \geqq t^{*}$,

$$
\sup _{s \leqq t}\left\|\varphi_{v}(s)-\varphi(s)\right\|_{L_{2}^{1}} \rightarrow 0
$$


as $v \rightarrow \infty$, and

$$
\begin{aligned}
& \int_{t^{*}}^{t} k(t-s)\left\|\varphi_{v}(s)-\varphi(s)\right\|_{L_{8}} d s \\
& \quad \leqq C \sup _{s \leqq t}\left\|\varphi_{v}(s)-\varphi(s)\right\|_{L_{2}^{1}}+C\left(\int_{t^{*}}^{t}\left(\left\|\varphi_{0, v}(s)-\varphi_{0}(s)\right\|_{L_{8}}\right)^{4} d s\right)^{1 / 4}
\end{aligned}
$$

The estimates (22) and (23) imply (21).

By hyperbolicity and uniqueness of distributional solutions of (1), we have $\varphi_{v}(s)=\varphi(s)$ for $|x| \leqq R_{v}-s$. Hence (22) follows from the conservation of energy and

$$
\left\|\varphi_{v}(s)-\varphi(s)\right\|_{L_{2}^{1}} \leqq C\left(\left\|\varphi_{v}(s)\right\|_{L_{2}^{1}\left(|x| \geqq R_{v}-s\right)}+\|\varphi(s)\|_{L_{2}^{1}\left(|x| \geqq R_{v}-s\right)}\right) .
$$

Furthermore

$$
\begin{aligned}
\left\|\varphi_{v}(s)-\varphi(s)\right\|_{L_{8}} \leqq & C\left\|\varphi_{0, v}(s)-\varphi_{0}(s)\right\|_{L_{8}} \\
& +C \int_{0}^{s} k(s-\sigma)\left(\left(\left\|\varphi_{v}(\sigma)\right\|_{L_{2}^{1}}\right)^{2}+\left(\|\varphi(\sigma)\|_{L_{2}^{1}}\right)^{2}\right)\left\|\varphi_{v}(\sigma)-\varphi(\sigma)\right\|_{L_{8}} d \sigma \\
& +C \int_{0}^{s} k(s-\sigma)\left(\left\|\varphi_{v}(\sigma)\right\|_{L_{8}}+\|\varphi(\sigma)\|_{L_{8}}\right)\left(\left\|\varphi_{v}(\sigma)-\varphi(\sigma)\right\|_{L_{2}^{1}}\right)^{2} d \sigma .
\end{aligned}
$$

Lemma 4.5 gives

$$
\begin{aligned}
\left\|\varphi_{v}(s)-\varphi(s)\right\|_{L_{8}} \leqq & C\left\|\varphi_{0, v}(s)-\varphi_{0}(s)\right\|_{L_{8}}+C(u) \int_{0}^{s} k(s-\sigma)\left\|\varphi_{v}(\sigma)-\varphi(\sigma)\right\|_{L_{8}} d \sigma \\
& +C(u) \sup _{\sigma \leqq s}\left(\left\|\varphi_{v}(\sigma)-\varphi(\sigma)\right\|_{L_{2}^{1}}\right)^{2},
\end{aligned}
$$

$C(u)$ independent of $v$, and so, by Gronwall's inequality, there exists some function $h(s) \in L_{\infty}^{\text {loc }}$ such that

$$
\left\|\varphi_{v}(s)-\varphi(s)\right\|_{L_{8}} \leqq h(s)\left(\left\|\varphi_{0, v}(s)-\varphi_{0}(s)\right\|_{L_{8}}+\sup _{\sigma \leqq s}\left\|\varphi_{v}(\sigma)-\varphi(\sigma)\right\|_{L_{2}^{1}}\right)
$$

Multiply by $k(t-s)$ and integrate over $\left(t^{*}, t\right)$. Equation (23) follows, since $k \in L_{4 / 3}$. This completes the proof of Lemma 4.6.

We can now prove

$$
\sup _{u \in D}\|\varphi(u)\|_{L_{4}\left(L_{8}\right)}<\infty
$$

with a Gronwall technique applying the uniform weak decay estimate that is obtained in Lemma 4.6.

Set

and

$$
U_{0}(t)=\left\|\varphi_{0}(t)\right\|_{L_{8}}
$$

$$
U(t)=\|\varphi(t)\|_{L_{8}} .
$$

In view of Proposition 3.1 there exists an $\alpha>0$ such that

$$
k(t) \leqq C t^{-1-\alpha}, \quad t \geqq 1 .
$$

From Lemma 4.3 there exists a constant $C(D)$, independent of $t$ and $\eta$ such that 
for $0 \leqq|\eta| \leqq \frac{1}{2}$,

$$
\left\|\varphi^{3}(t)\right\|_{L_{8 / 7}^{1}} \leqq C(D)\left(\|\varphi(t)\|_{L_{8}}\right)^{1+\eta}
$$

Hence we conclude that

$$
U(t) \leqq U_{0}(t)+C(D) \int_{0}^{t} k(t-s) U(s)^{1+\eta} d s
$$

for $0 \leqq|\eta| \leqq \frac{1}{2}$. Lemma 4.5 yields

$$
U(s) \leqq U_{0}(s)+C(D)
$$

for $s \in \mathbf{R}$. In particular, by Lemma $4.4, U \in L_{4}([0, \tilde{t}])$, uniformly for $u \in D$, for $\tilde{t}<\infty$. It remains to show that $U \in L_{4}([\tilde{t}, \infty))$, uniformly in $u \in D$, for some suitable chosen $\tilde{t}$.

So let $t^{*}$ be large (to be determined later) and let us inductively define $U_{j}$, $j=1,2, \ldots, N$, where $N$ is the smallest integer such that $N \alpha \geqq 1+\alpha$ satisfying

$$
U(s) \leqq U_{j-1}(s)+C\left(t^{*}\right)\left((1+s)^{-\alpha(j-1)}+(1+s)^{-1-\alpha}\right), \quad 2^{j} t^{*} \leqq s .
$$

Insert (25) and (26) in (24). For $t \geqq 2^{j+1} t^{*}$ we obtain

$$
\begin{aligned}
U(t) \leqq & U_{0}(t)+C(D)\left\{\int_{0}^{2 j^{*}} k(t-s) U(s)^{\eta} U_{0}(s) d s\right. \\
& +\int_{0}^{2 j t^{*}} k(t-s) d s+\int_{2^{j} t^{*}}^{t / 2} k(t-s) U(s)^{\eta} U_{j-1}(s) d s \\
& \left.+\int_{2^{j} t^{*}}^{t / 2} k(t-s)\left((1+s)^{-\alpha(j-1)}+(1+s)^{-1-\alpha}\right) d s+\int_{t / 2}^{t} k(t-s) U(s)^{1+\eta} d s\right\} .
\end{aligned}
$$

Now introduce the operators $K_{j 1}, K_{j 2}, K_{j 3}$ depending on $t^{*}, \eta$ by

$$
\begin{aligned}
& K_{j 1} g(t)=\int_{2^{j} j^{*}}^{t / 2} k(t-s) U(s)^{n} g(s) d s, \\
& K_{j 2} g(t)=\int_{0}^{2 j t^{*}} k(t-s) U(s)^{n} g(s) d s
\end{aligned}
$$

and

$$
K_{j 3} g(t)=\left(\int_{2 t^{*}}^{t} k(t-s) g(s)^{1 /(1-\eta)} d s\right)^{1-\eta} .
$$

Lemma 4.5 implies that

$$
\begin{aligned}
K_{j 1} g(t) \leqq & C\left(\int_{2 j t^{*}}^{t} k(t-s) U(s) d s\right)^{\eta} \\
& \cdot\left(\int_{2 j_{t^{*}}}^{t} k(t-s) g(s)^{1 /(1-\eta)} d s\right)^{1-\eta} \leqq C(D) K_{j 3} g(t) .
\end{aligned}
$$


This yields

$$
\begin{aligned}
\left\|K_{j 1} g\right\|_{L_{4}([\omega, \infty))} & \leqq C(D)\left\|K_{j 3} g\right\|_{L_{4}([\omega, \infty))} \\
& \leqq C(D)\left(\int_{0}^{\infty} k(s) d s\right)^{1-\eta}\|g\|_{L_{4}\left(\left[2^{j} t^{*}, \infty\right)\right)} \leqq C(D)\|g\|_{L_{4}\left(\left[2^{j} t^{*}, \infty\right)\right)},
\end{aligned}
$$

for $\omega \geqq 2^{j+1} t^{*}$, with $C(D)$ independent of $j, t^{*}, \omega$. In particular, the operators $K_{j 1}, K_{j 2}, K_{j 3}$ are bounded on $L_{4}([\omega, \infty)), \omega \geqq 2^{j+1} t^{*}$ with operator norms bounded independently of $j, \omega$ and $t^{*}$ and boundedly dependent on $\|u\|_{\mathbf{X}}$. Moreover we have

$$
\begin{gathered}
\int_{0}^{2 j t^{*}} k(t-s) d s \leqq C 2^{j} t^{*}(1+t)^{-1-\alpha}, \\
\int_{2^{j} t^{*}}^{t / 2} k(t-s)\left((1+s)^{-\alpha(j-1)}+(1+s)^{-1-\alpha}\right) d s \leqq C\left\{(1+t)^{-1-\alpha}+(1+t)^{-\alpha j}\right\}
\end{gathered}
$$

and

$$
\begin{aligned}
\int_{t / 2}^{t} k(t-s) U(s)^{1+\eta} d s \leqq & \left(\int_{t / 2}^{t} k(t-s) U(s)^{1 /(1-\eta)} d s\right)^{1-\eta} \\
& \cdot\left(\int_{t / 2}^{t} k(t-s) U(s) d s\right)^{\eta} \leqq \varepsilon\left(t^{*}\right) K_{j 3} U(t),
\end{aligned}
$$

where $\varepsilon\left(t^{*}\right) \rightarrow 0$ as $t^{*} \rightarrow \infty$, uniformly for $u \in D$ by Lemma 4.6 . Hence for $t \geqq 2^{j+1} t^{*}$ we have

$$
\begin{aligned}
U(t) \leqq & U_{0}(t)+C(D)\left\{K_{j 2} U_{0}(t)+K_{j 1} U_{j-1}(t)\right. \\
& \left.+C\left(t^{*}\right)\left((1+t)^{-\alpha j}+(1+t)^{-1-\alpha}\right)+\varepsilon\left(t^{*}\right) K_{j 3} U(t)\right\}
\end{aligned}
$$

For $j=1,2, \ldots, N$, set

$$
U_{j}(t):=U_{0}(t)+C(D)\left\{K_{j 2} U_{0}(t)+K_{j 1} U_{j-1}(t)+\varepsilon\left(t^{*}\right) K_{j 3} U(t)\right\} .
$$

Hence

$$
\begin{aligned}
U_{j}(t)= & U_{0}(t)+\sum_{l=0}^{j-1} C(D)^{l+1} K_{j 1} K_{(j-1) 1} \cdots K_{(j-l) 1} U_{0}(t) \\
& +C(D) K_{j 2} U_{0}(t)+\sum_{l=0}^{j-2} C(D)^{l+2} K_{j 1} K_{(j-1) 1} \cdots K_{(j-l) 1} K_{(j-l-1) 2} U_{0}(t) \\
& +\varepsilon\left(t^{*}\right)\left\{C(D) K_{j 3} U(t)+\sum_{l=0}^{j-2} C(D)^{l+2} K_{j 1} K_{(j-1) 1} \cdots K_{(j-l) 1} K_{(j-l-1) 3} U(t)\right\}
\end{aligned}
$$

for $j=2,3, \ldots, N$. Then choose $t^{*}$ sufficiently large such that for $L_{4}:=L_{4}\left(\left[2^{N+1} t^{*}, \infty\right)\right)$, $\varepsilon\left(t^{*}\right)\left\|C(D) K_{j 3} U+\sum_{l=0}^{j-2} C(D)^{l+2} K_{j 1} K_{(j-1) 1} \cdots K_{(j-l) 1} K_{(j-l-1) 3} U\right\|_{L_{4}} \leqq \frac{1}{2}\|U\|_{L_{4}}$

Since $K_{j 1}, K_{j 2}, j=1,2, \ldots, N$ are bounded operators and $(1+t)^{-1-\alpha} \in L_{4}$ we obtain

$$
\frac{1}{2}\|U\|_{L_{4}} \leqq C\left\|U_{0}\right\|_{L_{4}}+C \text {. }
$$


Finally choosing $\tilde{t}=2^{N+1} t^{*}$ completes the proof of

$$
\sup _{u \in D}\|\psi(u)\|_{L_{4}\left([0, \infty) ; L_{8}\right)}<\infty .
$$

By reversing time we prove

$$
\sup _{u \in D}\|\psi(u)\|_{L_{4}\left((-\infty, 0] ; L_{8}\right)}<\infty
$$

which ends the proof of Lemma 4.2.

\section{References}

1. Baez, J.: Scattering and complete integrability in conformally invariant nonlinear theories. J. Math. Phys. 31, 757-762 (1990)

2. Baez, J., Zhou, Z.: Scattering and complete integrability in the massive $\varphi^{4}$ theory, preprint

3. Baez, J., Zhou, Z.: Analyticity of scattering for the $\varphi^{4}$ theory. Commun. Math. Phys. 124, 9-21 (1989)

4. Brenner, $\mathrm{Ph} .:$ On scattering and everywhere defined scattering operators for nonlinear KleinGordon equation. J. Diff. Eq. 56, 310-344 (1985)

5. Brenner, $\mathrm{Ph} .:$ On space-time means and everywhere defined scattering operators for nonlinear Klein-Gordon equations. Math Z. 186, 383-391 (1984)

6. Brenner, Ph.: On strong global solutions of nonlinear hyperbolic equations, Seminaire equations aux derivees partielles 1988-1989 Ecole polytechnique. Centre de mathematiques

7. Gilbarg, D., Trudinger, N. S.: Elliptic partial differential equations of second order. Berlin, Heidelberg, New York: Springer 1983

8. Hille, E., Phillips, R. S.: Functional analysis and semigroups. American Mathematical Society, Colloquium Publications, Vol. XXXI, 1957

9. Marshall, B.: Mixed norm estimates for the Klein-Gordon equation. In: Conference on harmonic analysis in honor of Antoni Zygmund, Vol. 2, Wadsworth Math. Ser. Blemont, California: Wadsworth 1983, pp. 638-649

10. Marshall, B., Strauss, W., Wainger, S.: $L^{p}-L^{q}$ estimates for the Klein-Gordon equation. J. Math. Pures Appl. 59, 417-440 (1980)

11. Morawetz, C. S., Strauss, W.: Decay and scattering of solutions of a nonlinear relativistic wave equation. Commun. Pure Appl. Math. 25, 1-31 (1972)

12. Strauss, W.: Nonlinear scattering theory at low energy. J. Funct. Anal. 41, 110-133 (1981)

Communicated by A. Jaffe 